Claremont Colleges

Scholarship@ Claremont

All HMC Faculty Publications and Research

HMC Faculty Scholarship

$1-1-2006$

\title{
Reflections Acting Efficiently on a Building
}

Michael E. Orrison

Harvey Mudd College

\section{Recommended Citation}

Orrison, Michael E. "Reflections Acting Efficiently on a Building." Forum Mathematicum 18.3 (2006): 419-25. Print.

This Article - preprint is brought to you for free and open access by the HMC Faculty Scholarship at Scholarship @ Claremont. It has been accepted for inclusion in All HMC Faculty Publications and Research by an authorized administrator of Scholarship @ Claremont. For more information, please contact scholarship@cuc.claremont.edu. 
This article appeared in Forum Mathematicum 18 (2006), no. 3, 419-425.

\title{
REFLECTIONS ACTING EFFICIENTLY ON A BUILDING
}

\author{
MICHAEL E. ORRISON
}

\begin{abstract}
We show how Radon transforms may be used to apply efficiently the class sum of reflections in the finite general linear group $G L_{n}\left(\mathbb{F}_{q}\right)$ to vectors in permutation modules arising from the action of $G L_{n}\left(\mathbb{F}_{q}\right)$ on the building of type $A_{n-1}\left(\mathbb{F}_{q}\right)$.
\end{abstract}

\section{INTRODUCTION}

Let $G$ be a finite group acting transitively on a finite set $X$, and let $M$ be the resulting $\mathbb{C}[G]$ permutation module. If $C$ is a conjugacy class in $G$, then its class sum $T=\sum_{c \in C} c \in \mathbb{C}[G]$ may be viewed as a diagonalizable linear transformation $T: M \rightarrow M$. The question addressed in this paper is the following:

Given $f \in M$, how may we compute $T f$ efficiently?

This question arises in spectral analysis, which is a non-model based approach to the analysis of data arising as a complex-valued function $f$ on a set $X$ with a group $G$ of automorphisms. Developed by Diaconis in $[6,7]$, the subject extends the classical spectral analysis of time series and requires the projection of $f$ onto $\mathbb{C}[G]$-invariant subspaces of $M$.

Class sums play an important role in spectral analysis because their eigenspaces are direct sums of fundamental invariant subspaces known as isotypic subspaces or homogeneous components. With a suitable collection of class sums, isotypic projections may be achieved as eigenspace projections, and being able to apply class sums efficiently allows us to make effective use of iterative eigenspace projection techniques $[10,11]$.

In this paper, we use Radon transforms to show how the class sum $T$ of reflections in the finite general linear group may be applied surprisingly efficiently (see Theorem 6 and Theorem 8) when the underlying set is a set of residues of the building of type $A_{n-1}\left(\mathbb{F}_{q}\right)$. Our work builds directly upon that found in [12] in which Radon transforms were used to show that the eigenspaces of $T$ are precisely the isotypic subspaces of such permutation modules. It also extends some of the ideas found in [10] and [11]. See [1,2] for different examples of Radon transforms associated with buildings.

We assume the reader is familiar with buildings and chamber systems. More specifically, we assume the reader is familiar with the building of type $A_{n-1}\left(\mathbb{F}_{q}\right)$ whose chambers may be viewed as maximal flags in an $n$-dimensional vector space

2000 Mathematics Subject Classification. 20E42, 20C40, 43A85.

Key words and phrases. Building, Radon transform, reflection. 
over the finite field $\mathbb{F}_{q}$ with $q$ elements, and whose corresponding Weyl group is the symmetric group $S_{n}$ (see, for example, $[4,13]$ ).

\section{BACKGROUND}

In this section, we use incidence relations to define Radon transforms, and describe the computational model we use to compare different approaches to applying a fixed linear transformation. We also review some terminology related to buildings, and discuss the class sum of reflections in the finite general linear group.

Radon Transforms. Let $G$ be a finite group acting on finite sets $X$ and $Y$, and let $M$ and $N$ be the resulting $\mathbb{C}[G]$ permutation modules, respectively (see, for example, [14]). We say that the elements of $X$, when thought of as vectors in $M$, form the usual basis of $M$.

Suppose there is an incidence relation between $X$ and $Y$. We write $x \sim y$ if $x \in X$ is incident to $y \in Y$, and define the Radon transform (see [3]) $R: M \rightarrow N$ by setting

$$
R(x)=\sum_{y: x \sim y} y
$$

The adjoint $R^{*}: N \rightarrow M$ is defined by setting

$$
R^{*}(y)=\sum_{x: x \sim y} x
$$

Thus, if the incidence relation is invariant under the action of $G$, then $R, R^{*}$ and $R^{*} R$ are $\mathbb{C}[G]$-homomorphisms.

Computational Model. As in [8], to compare different approaches to applying a fixed linear transformation, we use a computational model that counts one complex multiplication followed by one complex addition as one operation. Since a linear transformation $T$ on a permutation module $M$ may be viewed as a matrix with respect to the usual basis of $M$, the number of operations needed to apply $T$ is never more than the number of nonzero entries in its corresponding matrix.

The Building of Type $A_{n-1}\left(\mathbb{F}_{q}\right)$. We now turn our attention to the action of the finite general linear group on the residues of the building of type $A_{n-1}\left(\mathbb{F}_{q}\right)$. See [4] or [13] for the relevant background on buildings. Note that, for convenience, we assume $q \neq 2$. The case $q=2$ is, however, computationally similar. See [12] for details.

Let $V$ be an $n>1$ dimensional vector space over the finite field $\mathbb{F}_{q}$ with $q$ elements, and let $G L_{n}\left(\mathbb{F}_{q}\right)=G_{n}$ be the group of automorphisms of $V$. Recall that the chambers of the building $\Delta$ of type $A_{n-1}\left(\mathbb{F}_{q}\right)$ may be viewed as nested sequences of subspaces, or flags,

$$
V_{0} \supset \cdots \supset V_{n}
$$

where $V_{i}$ is a subspace of $V$ of dimension $n-i$. Let $I=\{1, \ldots, n-1\}$. If $i \in I$, then two chambers $V_{0} \supset \cdots \supset V_{n}$ and $V_{0}^{\prime} \supset \cdots \supset V_{n}^{\prime}$ are said to be $i$-adjacent if $V_{j}=V_{j}^{\prime}$ for all $j \neq i$. This gives rise to a chamber system over $I$. Residues of cotype $J=\left\{j_{1}, \ldots, j_{m-1}\right\} \subset I$ where $j_{i}<j_{i+1}$ may then be viewed as flags

$$
V_{0} \supset \cdots \supset V_{m}
$$


where $V_{0}=V, V_{m}=0$, and $V_{i}$ is a subspace of dimension $d_{i}=n-j_{i}$ when $0<i<m$. For convenience, we say that the flag $V_{0} \supset \cdots \supset V_{m}$ is of type $\lambda=\left(\lambda_{1}, \ldots, \lambda_{m}\right)$ where $\lambda_{i}=d_{i-1}-d_{i}$. For example, chambers correspond to flags of type $(1, \ldots, 1)$.

Let $X_{\lambda}$ denote the set of flags of type $\lambda$. The action of $G_{n}$ on $V$ gives rise to a transitive action of $G_{n}$ on $X_{\lambda}$, and we denote the corresponding $\mathbb{C}\left[G_{n}\right]$ permutation module by $M_{\lambda}$. Note that for any sequence $\mu=\left(\mu_{1}, \ldots, \mu_{l}\right)$ of non-negative integers whose sum is $n$, there is a corresponding set of flags $X_{\mu}$ of type $\mu$ and a permutation module $M_{\mu}$.

If $b_{1}, \ldots, b_{n}$ are linearly independent vectors in $V$, let $\left(b_{1}, \ldots, b_{n}\right)$ denote the chamber

$$
\left\langle b_{1}, \ldots, b_{n}\right\rangle \supset\left\langle b_{2}, \ldots, b_{n}\right\rangle \supset \cdots \supset\left\langle b_{n}\right\rangle \supset 0 .
$$

By fixing a basis $e_{1}, \ldots, e_{n}$ of $V$, we create the fundamental apartment $\Sigma$ of $\Delta$ by taking nested sequences of subspaces spanned by subsets of $e_{1}, \ldots, e_{n}$. The chambers of $\Sigma$ are therefore those maximal flags $\left(e_{\sigma(1)}, \ldots, e_{\sigma(n)}\right)$ where $\sigma$ ranges over the symmetric group $S_{n}$.

Recall that the Weyl group associated to $\Delta$ is also $S_{n}$ and that we therefore have an $S_{n}$-distance function

$$
\delta: \Delta \times \Delta \rightarrow S_{n} .
$$

For example, if $x=\left(e_{1}, \ldots, e_{n}\right)$ and $y=\left(e_{\sigma(1)}, \ldots, e_{\sigma(n)}\right)$, then $\delta(x, y)=\sigma$. Thus if the chambers $x$ and $y$ are $i$-adjacent, then $\delta(x, y)$ is the transposition $(i, i+1)$.

The Class Sum of Reflections. The fundamental reflections of $\Sigma$ are those automorphisms $s_{1}, \ldots, s_{n-1}$ where $s_{i}$ exchanges the basis vectors $e_{i}$ and $e_{i+1}$ while leaving the other basis vectors fixed. The $s_{i}$ are conjugate to each other in $G_{n}$ and are therefore contained in the same conjugacy class $C$. Furthermore, each $c \in C$ fixes a hyperplane (codimension-1 subspace) of $V$ pointwise. For example, $s_{1}$ fixes the hyperplane

$$
\left\langle e_{1}+e_{2}, e_{3}, e_{4}, \ldots, e_{n}\right\rangle
$$

pointwise. We refer to $C$ as the conjugacy class of reflections and denote the class sum of $C$ by $T$.

Now if $x$ is the (not necessarily maximal) flag $V_{0} \supset \cdots \supset V_{m}$, then $x$ determines a partition $\left\{P_{1}(x), \ldots, P_{m}(x)\right\}$ of the hyperplanes of $V$ where we say the hyperplane $H$ is in $P_{j}(x)$ if $H$ contains $V_{j}$ but not $V_{j-1}$. We will make use of this partition in this and the next section.

Let $c$ be a reflection and suppose that $c$ fixes the hyperplane $H \in P_{j}(x)$ pointwise. Fix $v \in V_{j-1}-\left(H \cap V_{j-1}\right)$. The vectors $v$ and $c v$ are exchanged by $c$ since $c^{2}$ is the identity. It follows that $v+c v \in H$, thus $c v=-v+h$ for some $h \in H$.

Suppose $c v$ is contained in $V_{i-1}$ but not $V_{i}$. Notice that $i \leq j$ and that each vector in $V_{j-1}-\left(H \cap V_{j-1}\right)$ will yield the same $i$. Define $\varphi(x, c)=(i, j)$. It is easy to show that $x=c x$ if and only if $i=j$, and that if $i<j$, then $x \cap c x=\left(V_{0} \cap c V_{0}\right) \supseteq$ $\cdots \supseteq\left(V_{m} \cap c V_{m}\right)$ is a flag of type $\mu=\left(\mu_{1}, \ldots, \mu_{m}\right)$ where $\mu_{i}=\lambda_{i}+1, \mu_{j}=\lambda_{j}-1$, and $\mu_{k}=\lambda_{k}$ for $k \neq i, j$.

Thus, if $i<j$, we may define $T_{i j}: M_{\lambda} \rightarrow M_{\lambda}$ by setting $T_{i j}(x)=\sum y$ where the sum is over all $y$ such that $y=c x$ for some reflection $c$ where $\varphi(x, c)=(i, j)$.

If $k$ is a non-negative integer, define $[k]=1+q+q^{2}+\cdots+q^{k-1}$. We then have the following lemmas. Proofs may be found in [12]. 
Lemma 1. Let $x \in X_{\lambda}$. If $1 \leq i<j \leq m$, then there are $q^{2\left(d_{i}-d_{j-1}\right)+1}\left[\lambda_{i}\right]\left[\lambda_{j}\right]$ flags $y$ such that $y=c x$ for some reflection $c$ where $\varphi(x, c)=(i, j)$.

Lemma 2. If $T$ is viewed as a linear transformation $T: M_{\lambda} \rightarrow M_{\lambda}$, then

$$
T=\sum_{1 \leq i<j \leq m} q^{n-2+d_{j-1}-d_{i}}(q-1) T_{i j}+\left(\sum_{j=1}^{m} q^{n-1}\left[\lambda_{j}\right]\right) I .
$$

\section{Applying the Class Sum of Reflections}

We now turn our attention to applying the class sum $T$ of reflections to arbitrary vectors in $M_{\lambda}$. We begin by considering the direct application of $T$ when viewed as a matrix with respect to the usual basis of $M_{\lambda}$.

Proposition 3. The number of operations needed to apply $T$ to an arbitrary vector in $M_{\lambda}$ is no more than

$$
\left(1+\sum_{1 \leq i<j \leq m} q^{2\left(d_{i}-d_{j-1}\right)+1}\left[\lambda_{i}\right]\left[\lambda_{j}\right]\right)\left|X_{\lambda}\right| .
$$

Proof. When viewed as a matrix with respect to the usual basis of $M_{\lambda}$, each column of $T$ contains

$$
1+\sum_{1 \leq i<j \leq m} q^{2\left(d_{i}-d_{j-1}\right)+1}\left[\lambda_{i}\right]\left[\lambda_{j}\right]
$$

nonzero entries by Lemma 1 and Lemma 2 . The proposition follows immediately.

Using Radon Transforms to Apply T. As noted in the introduction, Proposition 3 may be improved upon by writing $T$ as a linear combination of related Radon transforms.

Let $x$ be the flag $V_{0} \supset \cdots \supset V_{m}$ of type $\lambda$. Let $1 \leq i<j \leq m$ and let $\mu=\left(\mu_{1}, \ldots, \mu_{m}\right)$ where $\mu_{i}=\lambda_{i}+1, \mu_{j}=\lambda_{j}-1$, and $\mu_{k}=\lambda_{k}$ for $k \neq i, j$. We say that $x$ is $i j$-incident to $y \in X_{\mu}$ if $y$ is a flag of the form

$$
V_{0} \supset \cdots \supset V_{i-1} \supset H \cap V_{i} \supset \cdots \supset H \cap V_{j-1} \supseteq V_{j} \supset \cdots \supset V_{m}
$$

for some $H \in P_{j}(x)$. This incidence relation is invariant under the action of $G_{n}$, thus the associated Radon transform $R_{i j}: M_{\lambda} \rightarrow M_{\mu}$ is a $\mathbb{C}\left[G_{n}\right]$-homomorphism.

The following is Theorem 13 in [12]:

Theorem 4. If $T$ is viewed as a linear transformation $T: M_{\lambda} \rightarrow M_{\lambda}$, then

$$
T=\sum_{1 \leq i<j \leq m} q^{n-1+d_{j-1}-d_{i}-i}(q-1) R_{i j}^{*} R_{i j}+\left(\sum_{j=1}^{m} q^{n-j}\left[\lambda_{j}\right]\right) I .
$$

We may therefore apply $T$ using the $R_{i j}$ and $R_{i j}^{*}$.

Lemma 5. The number of operations needed to apply $R_{i j}$ or $R_{i j}^{*}$ is no more than

$$
q^{d_{i}-d_{j-1}}\left[\lambda_{j}\right]\left|X_{\lambda}\right| \text {. }
$$

Proof. By Lemma 5 in [12], each flag in $M_{\lambda}$ is $i j$-incident to $q^{d_{i}-d_{j-1}}\left[\lambda_{j}\right]$ flags in $M_{\mu}$. Thus, when viewed as a matrix with respect to the usual basis, each column of $R_{i j}$ contains $q^{d_{i}-d_{j-1}}\left[\lambda_{j}\right]$ nonzero entries. Since $R_{i j}^{*}$ is the transpose of $R_{i j}$, the lemma follows. 
Given Lemma 5, we may now state the following theorem, which should be compared to Proposition 3:

Theorem 6. The number of operations needed to apply $T$ to an arbitrary vector in $M_{\lambda}$ is no more than

$$
\left(\frac{m^{2}-m+2}{2}+\sum_{1 \leq i<j \leq m} 2 q^{d_{i}-d_{j-1}}\left[\lambda_{j}\right]\right)\left|X_{\lambda}\right| .
$$

Proof. By Lemma 5, the number of operations needed to apply a scalar multiple of $R_{i j}^{*} R_{i j}$ is no more than

$$
\left(1+2 q^{d_{i}-d_{j-1}}\left[\lambda_{j}\right]\right)\left|X_{\lambda}\right|
$$

Thus, by Theorem 4 , the number of operations needed to apply $T$ is no more than

$$
\begin{aligned}
\left(1+\sum_{1 \leq i<j \leq m}\left(1+2 q^{d_{i}-d_{j-1}}\left[\lambda_{j}\right]\right)\right)\left|X_{\lambda}\right|= & \\
& \left(\frac{m^{2}-m+2}{2}+\sum_{1 \leq i<j \leq m} 2 q^{d_{i}-d_{j-1}}\left[\lambda_{j}\right]\right)\left|X_{\lambda}\right| .
\end{aligned}
$$

Restricting to Chambers. We now restrict our attention to the action of $G_{n}$ on the chambers of $\Delta$. For convenience, let $X$ denote the set $X_{(1, \ldots, 1)}$ of chambers and let $M$ denote the resulting $\mathbb{C}\left[G_{n}\right]$-module $M_{(1, \ldots, 1)}$.

When viewed as a matrix with respect to the usual basis of $M$, the number of operations needed to directly apply $T$ to an arbitrary vector in $M$ is no more than

$$
\left(1+\sum_{1 \leq i<j \leq n} q^{2(j-i)-1}\right)|X|
$$

by Proposition 3. By Theorem 6, this number may be improved to

$$
\left(\frac{n^{2}-n+2}{2}+\sum_{1 \leq i<j \leq n} 2 q^{(j-i)-1}\right)|X|
$$

by using Radon transforms. This bound may be improved even further by taking into account the relationship between reduced galleries and the $S_{n}$-distance function $\delta$.

Lemma 7. Let $c$ be a reflection and let $x$ be a chamber. If $x \neq c x$ and $\varphi(x, c)=$ $(i, j)$, then $\delta(x, c x)$ is the transposition $(i, j) \in S_{n}$.

Proof. Let $H$ be the hyperplane fixed pointwise by $c$. It is easy to show that if $\varphi(x, c)=(i, j)$ and $i \neq j$, then there is a basis $b_{1}, \ldots, b_{n}$ of $V$ such that $x=$ $\left(b_{1}, \ldots, b_{n}\right), c$ exchanges $b_{i}$ and $b_{j}$, and $b_{k} \in H$ if $k \neq i, j$. It follows that $\delta(x, c x)$ is the transposition $(i, j)$.

We may now take advantage of the relationship between reduced galleries in $\Delta$ and the $S_{n}$-distance function $\delta: \Delta \times \Delta \rightarrow S_{n}$ to show how $T$ may be applied with a surprisingly small number of operations. In particular, the following bound replaces the scalar $\left(n^{2}-n+2\right) / 2+\sum_{1 \leq i<j \leq n} 2 q^{(j-i)-1}$ in (1) with the much smaller $n^{3}$. 
Theorem 8. The number of operations required to apply $T: M \rightarrow M$ is less than

$$
n^{3}|X| \text {. }
$$

Proof. By Lemma 7, $T_{i j}: M \rightarrow M$ may now be defined by setting $T_{i j}(x)=\sum y$ where the sum is over all chambers $y$ such that $\delta(x, y)=(i, j)$. The transposition $(i, j)$ may be written as a reduced product of adjacent transpositions:

$$
(i, j)=(i, i+1) \cdots(j-2, j-1)(j-1, j)(j-2, j-1) \cdots(i, i+1) .
$$

It follows that

$$
T_{i j}=T_{i, i+1} \cdots T_{j-2, j-1} T_{j-1, j} T_{j-2, j-1} \cdots T_{i, i+1} .
$$

By Lemma 12 in [12], $T_{i, i+1}=R_{i, i+1}^{*} R_{i, i+1}-I$. Thus, by Lemma $5, T_{i, i+1}$ may be applied using no more than $3|X|$ operations. A scalar multiple of $T_{i j}$ may therefore be applied using no more than

$$
|X|+(2(j-i)-1)(3|X|)=(6(j-i)-2)|X|
$$

operations. Hence, by Lemma 2 , the number of operations required to apply $T$ is no more than

$$
\left(1+\sum_{1 \leq i<j \leq n}(6(j-i)-2)\right)|X|<n^{3}|X| .
$$

\section{REFERENCES}

[1] Atanasi, L.: Radon transform on affine buildings of rank 3. J. Austral. Math. Soc. (Series A) 66 (1999), 66-89.

[2] Atanasi, L.: Radon transforms on $\widetilde{A}_{n}$-buildings. Math. Proc. Cambridge Philos. Soc. 128 (2000), no. 3, 425-439.

[3] Bolker, E.: The finite Radon transform. Contemporary Mathematics 63 (1987), 27-50.

[4] Brown, K.: Buildings. Springer-Verlag, New York 1989.

[5] Cartwright, D.: A brief introduction to buildings. Contemporary Mathematics 206 (1997), 45-77.

[6] Diaconis, P.: Group Representations in Probability and Statistics. Inst. of Math. Stat., Hayward, CA 1988.

[7] Diaconis, P.: A generalization of spectral analysis with applications to ranked data. Ann. of Stat. 17 (1989), no. 3, 949-979.

[8] Diaconis, P. and Rockmore, D.: Efficient computation of isotypic projections for the symmetric group. DIMACS series in discrete mathematics and theoretical computer science 11 (1993), 87-104.

[9] James, G.: Representations of General Linear Groups. Cambridge University Press, Cambridge 1984 .

[10] Maslen, D., Orrison, M. and Rockmore, D.: Computing isotypic projections with the Lanczos iteration. SIAM Journal on Matrix Analysis and Applications 25 (2004), no. 3, 784-803.

[11] Orrison, M.: An eigenspace approach to decomposing representations of finite groups. Ph.D. thesis, Dartmouth College, 2001.

[12] Orrison, M.: Radon transforms and the finite general linear groups. Forum Mathematicum, 16 (2004), 97-107.

[13] Ronan, M.: Lectures on Buildings. Academic Press, Inc., 1989.

[14] Serre, J.-P.: Linear Representations of Finite Groups. Springer-Verlag, New York-HeidelbergBerlin 1977.

Department of Mathematics, Harvey Mudd College, Claremont, CA 91711

E-mail address: orrison@hmc.edu 